\title{
An Efficient Instantaneous ECG Delineation Algorithm
}

\author{
Thion Ming Chieng ${ }^{1}$, Yuan Wen $\mathrm{Hau}^{2}$, Zaid Bin Omar ${ }^{3}$, Chiao Wen $\mathrm{Lim}^{4}$ \\ ${ }^{2}$ IJN-UTM Cardiovascular Engineering Centre, School of Biomedical Engineering and Health \\ Sciences, Faculty of Engineering, Universiti Teknologi Malaysia, Johor Bahru, Johor, Malaysia \\ ${ }^{3}$ School of Electrical Engineering, Faculty of Engineering, Universiti Teknologi Malaysia, Johor \\ Bahru, Johor, Malaysia \\ ${ }^{4}$ Faculty of Medicine, Universiti Teknologi MARA, Sungai Buloh, Selangor, Malaysia
}

\begin{abstract}
An efficient electrocardiogram (ECG) delineation algorithm is proposed to instantaneously delineate the ECG characteristic points, such as peak, onset and offset points of $Q R S, P$ and $T$ waves. It is essential to delineate the ECG characteristic waves accurately and precisely as it ensure the performance of ECG analysis and diagnosis. The proposed delineation algorithm is based on discrete wavelet transform (DWT) and moving window average (MWA) techniques. The proposed delineation algorithm is evaluated and assessed with the annotation data of $Q T$ database in term of accuracy, sensitivity and positive predictive value. With the only available 13 sets $Q T$ database records with modified Lead II data, the proposed algorithm achieved significant $P$ peak, $R$ peak, T peak and $T$ offset delineation performance with the accuracy of $95.34 \%, 99.80 \%, 90.82 \%$ and $86.33 \%$ respectively when evaluated with q1c annotation file. The mean difference between detected and annotated T offset based on qlc and $q 2 \mathrm{c}$ is $13 \mathrm{~ms}$ and $3.6 \mathrm{~ms}$ respectively. The delineation of 15 minute-long ECG record only required 74.702 second. As conclusion, the proposed ECG delineation algorithm based on DWT and MWA techniques have been proven simple, efficient and accurate in delineating the significant ECG characteristic points.
\end{abstract}

\section{Introduction}

Over the decades, electrocardiography has been used as a routine modality by cardiologists for cardiac assessment, monitoring and diagnosis due to its simplicity, cost effective and non-invasiveness characteristics. Electrocardiogram (ECG) is the biosignal acquired from electrocardiography by attaching the electrodes on the body surface of patient over time. ECG is a graphical representation of the propagation of electrical impulses triggered by cardiac muscles as it initiates the cardiac cycle. It is widely used to analyze and diagnose various cardiac disorders as ECG do conveys a huge amount of electrophysiological information that reflects the performance and health condition of the heart as well as its abnormalities [1, 2]. Normal sinus ECG consists of P wave, QRS complex, $T$ wave and conditional $U$ wave [3].

ECG delineation is the process of identifying the ECG characteristic points, such as peak, onset and offset points of ECG waves. Through delineation, clinical significant features can be derived and extracted from the morphology of the ECG characteristic wave, such as intervals, amplitude and shape of the wave. These features are used to access various structural and functional cardiac performance and assists the clinician in decision-making $[1,2]$. For instance, the abnormal prolongation or shortening of QT interval indicates the higher risk for the occurrence of ventricular arrhythmia and sudden cardiac death [4-6]. With clinician's manual visual inspection, ECG delineation and analysis can be a tough and tedious task as it requires a terrific amount of time and expert human resources [7-9]. The miss identification of complex and diverse ECG morphologies might occur during manual visual inspection. Hence, the development of efficient computerized ECG delineation approach is necessary as it plays a decisive role in diagnosing cardiac diseases.

In this study, we proposed a simple and efficient ECG delineation algorithm based on discrete wavelet transform and moving window average techniques to delineate the QRS complex, P and T waves. This article consists of four sections which begin with an introduction. Section 2 describes the methodology of proposed ECG delineation algorithm. Section 3 presents the performance evaluation of the delineation algorithm. Lastly, section 4 finalizes the article with the conclusion.

\section{Methodology}

This section describes the methodology of an efficient ECG delineation algorithm that instantaneously delineate the significant ECG characteristic waves. Figure 2 shows the overview workflow of the proposed ECG delineation algorithm based on discrete wavelet transform and moving 
window average techniques.

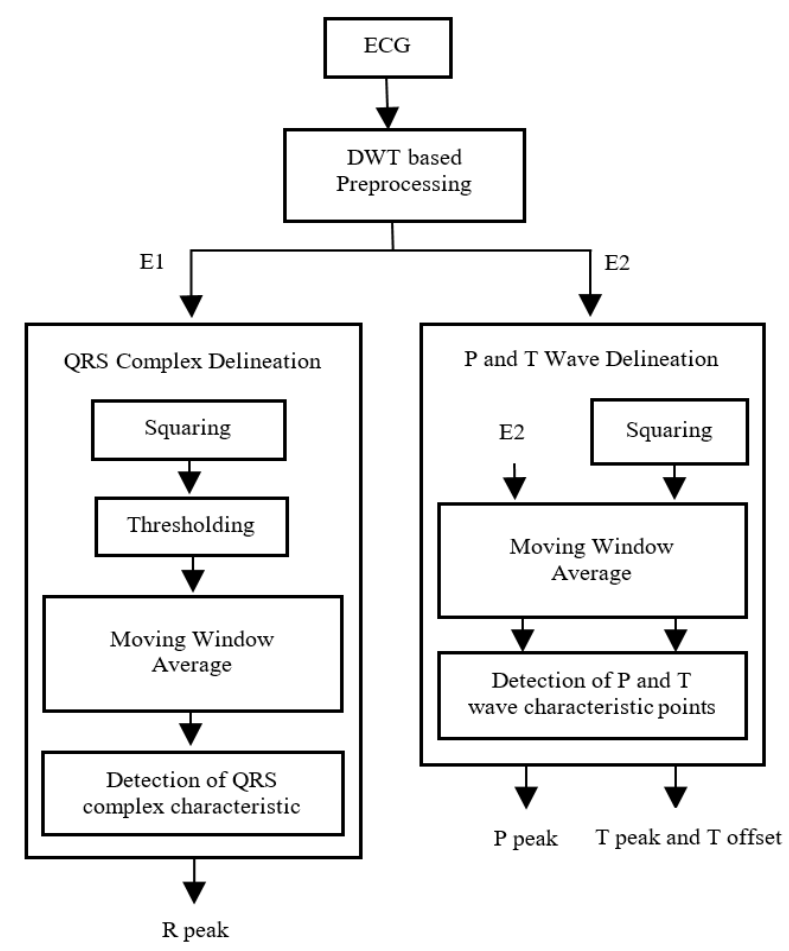

Figure 2. Workflow of the proposed ECG delineation algorithm

\subsection{ECG Datasets}

The ECG dataset used is QT Database [10] that acquired from PhysioNet [11]. It consists of 105 sets of fifteenminute ECG datasets with the sampling frequency of 250 $\mathrm{Hz}$. The proposed ECG delineation algorithm is designed based on the ECG Lead II morphology which has upright $\mathrm{P}, \mathrm{R}$ with the highest peak amplitude and $\mathrm{T}$ waves. QT Database consists of two independent annotation files which denoted as $q 1 c$ and $q 2 c$. Noted that some of the annotation data are not in continuous sequences of heartbeats and $\mathrm{P}$ wave annotation are unavailable in $q 2 c$ annotation file.

\subsection{Preprocessing}

ECG input signal is de-noises using notch filter with the cut-off frequency of $50 \mathrm{~Hz}$ to remove the powerline interference noise. Then, the baseline wandering and high frequency noises are de-noised using the discrete wavelet transform through the decomposition and reconstruction techniques. The wavelet decomposition level is defined at 12 level by the equation of Level of decomposition, $K={ }_{\log \frac{f s}{0.05}} / \log 2$ as the targeted ECG datasets are sampled at $250 \mathrm{~Hz}$. The mother wavelet of Daubechies 6 (db6) is used to decompose and reconstruct the ECG signal due to its structural similarity and filtering performance [12-14]. The filtered ECG signal can be acquired by applying the inverse wavelet transform to reconstruct the detailed coefficients with the frequency range of $0.05 \mathrm{~Hz}$ to $100 \mathrm{~Hz}$. Hence, the ECG artefacts are effectively eliminated while preserving the significant ECG features.

The filtered ECG are then isolated and separated into two sub-signals that comprises of QRS complex and P and $\mathrm{T}$ waves based on their frequency range. With the decomposition and reconstruction techniques, QRS complex with the frequency range of $10 \mathrm{~Hz}$ to $50 \mathrm{~Hz}$ [15] are isolated and separated from $\mathrm{P}$ and $\mathrm{T}$ waves with the frequency range of $0.6 \mathrm{~Hz}$ to $7 \mathrm{~Hz}$ [15] which denoted as E1 and E2 respectively as illustrated in Figure 2. This approach allows the efficient delineation of ECG characteristic wave without the need to introduce extra method for the removal of QRS complex, while, avoiding the possibility of miss identification of $\mathrm{R}$ peak and high $\mathrm{T}$ peak [16].

\section{ECG Delineation}

The workflow of the proposed ECG delineation algorithm are separated into two parts: QRS complex delineation and $\mathrm{P}$ and $\mathrm{T}$ wave delineation are shown in Figure 2. First, E1 signal is squared point by point, allows $\mathrm{R}$ peak become more significant and predominant wave. An adaptive threshold are implemented to reduce and eliminate those amplified ECG noises that did not contribute to QRS complex. The interest windows of QRS complex are generated based on two event-related moving window average technique which is firstly introduced by Elgendi [17]. The first moving window average, $\boldsymbol{M} \boldsymbol{W} \boldsymbol{A}_{Q R S}$ is used to extract the QRS features, while the second moving window average, $\boldsymbol{M} \boldsymbol{W} \boldsymbol{A}_{\text {beat }}$ is used to extract the ECG beat. Both moving window average are defined as the following equations 1 and 2.

$M W A_{Q R S}[n]=\frac{1}{w_{1}}\left(y\left[n-\frac{w_{1}-1}{2}\right]+\ldots+\mathrm{y}[\mathrm{n}]+\ldots+\mathrm{y}\left[\mathrm{n}+\frac{w_{1}-1}{2}\right]\right)$

Where $w_{1}$ is the window size of the first moving window average which defined as the approximate duration of QRS complex and rounded to the nearest odd integer.

$M W A_{\text {beat }}[n]=\frac{1}{w_{2}}\left(y\left[n-\frac{w_{2}-1}{2}\right]+\ldots+\mathrm{y}[\mathrm{n}]+\ldots+\mathrm{y}\left[\mathrm{n}+\frac{w_{2}-1}{2}\right]\right)$

Where $w_{2}$ is the window size of the second moving window average which defined as the approximate duration of a heartbeat and rounded to the nearest odd integer.

The optimal value for the window size of first and second moving window average is defined as 0.097 and 0.611 respectively [17]. The interest window of QRS 
complex are then identified as the region where the $M W A_{Q R S}$ is greater than $M W A_{\text {beat }}$ and set to 1.5 as depicted in equation 3 below. $R$ peak is then detected as the point with the maximum amplitude within the interest window of QRS complex.

$$
\text { Interest window of QRS complex }=\left\{\begin{array}{c}
1.5, M W A_{Q R S}>M W A_{\text {beat }} \\
0, \text { otherwise }
\end{array}\right.
$$

For identifying the interest window of $\mathrm{P}$ and $\mathrm{T}$ waves, similar approach are implemented. As the $\mathrm{P}$ wave is typically smaller than $\mathrm{T}$ wave, the window size of two moving window average is then based on the $\mathrm{P}$ wave duration [16]. The window size of first and second moving window average is 0.055 (half of the $\mathrm{P}$ wave duration) and 0.11 [16].The potential $P$ peak region is located on the left side of $R$ peak within the duration of one-third of successive R-R interval [18, 19]. Within potential $\mathrm{P}$ peak region, $\mathrm{P}$ peak is identify as the point with maximum amplitude inside the widest interest window of $\mathrm{P}$ wave.

For the $\mathrm{T}$ peak detection, similar procedures are repeated on the squared reconstructed signal, $E 2^{2}$ to avoid the miss detection of inverted $\mathrm{T}$ peak morphology. The potential $\mathrm{T}$ peak region located on right side of $\mathrm{R}$ peak within the duration of two-third of successive R-R interval $[18,19]$. $\mathrm{T}$ peak is identify as the point with maximum amplitude within the widest interest window of $\mathrm{T}$ wave in the potential $\mathrm{T}$ peak region. Once the $\mathrm{T}$ peak is identified, $\mathrm{T}$ offset are identify by the change of derivative sign based method. The $\mathrm{T}$ offset are identified as the point with the change of derivative sign from negative to positive for the normal upright $\mathrm{T}$ peak morphology. While, the change of derivative sign from positive to negative for the inverted $\mathrm{T}$ peak. The delineation of $\mathrm{P}, \mathrm{R}, \mathrm{T}$ peaks and $\mathrm{T}$ offset are illustrated in Figure 3.

\subsection{Performance Evaluation}

In this study, three statistical parameters, such as accuracy (Acc), sensitivity (Se) and positive predictive value (PPV) were calculated to evaluate the performance of the proposed instantaneous ECG delineation algorithm, which described in the following equations:

$$
\begin{gathered}
\text { Accuracy, } A C C=\frac{T P}{T P+F N+F P} \times 100 \% \\
\text { Sensitivity, } S e=\frac{T P}{T P+F N} \times 100 \% \\
\text { Positive Predictive Value, } P P V=\frac{T P}{T P+F P} \times 100 \%
\end{gathered}
$$

Where $T P$ is the true positive beat, $F N$ is the false negative beat and FP is the false positive beat.

The true positive (TP) beat is determined when the time difference between the annotated beat and detected beat is not greater than $150 \mathrm{~ms}$ which in obedience with
ANSI/AAMI-EC57:1998 standard [20]. Else, it is considered false positive (FP) as the time difference is greater than $150 \mathrm{~ms}$. The false negative $(\mathrm{FN})$ is found when there is no point detected within the range of $\pm 150 \mathrm{~ms}$ around annotated point. The performance of the proposed instantaneous ECG delineation algorithm is evaluated using the annotation files $q 1 c$ and $q 2 c$ as summarized in Table 1. The mean difference between detected and annotated $\mathrm{T}$ offset based on $q 1 c$ and $q 2 c$ is $13 \mathrm{~ms}$ and 3.6 $\mathrm{ms}$ respectively. In addition, the time required to delineate 15 minute-long ECG recording is measured which is about 74.702 second. Noted that the algorithm were executed in Matlab R2016a on a workstation with windows 10 operating system, Intel ${ }^{\circledR}$ Core $^{\mathrm{TM}} \mathrm{i} 7$ central processing unit at $3.40 \mathrm{GHz}$ and $16 \mathrm{~GB}$ of random-access memory.

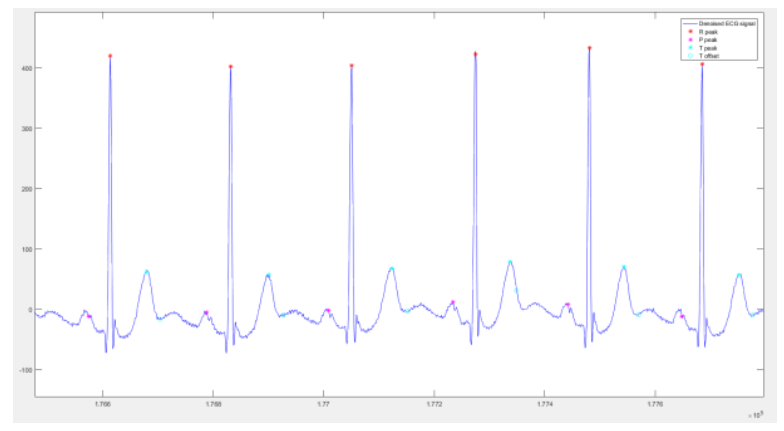

Figure 3. Delineation of P, R, T peaks and T offset.

Table 1. Performance evaluation of proposed instantaneous ECG delineation algorithm using the annotation file $q 1 c$ and $q 2 c$ of the QT database.

\begin{tabular}{ccccccccc}
\hline \multirow{2}{*}{ ECG } & \multicolumn{2}{c}{ P Peak } & \multicolumn{2}{c}{ R Peak } & \multicolumn{2}{c}{ T Peak } & \multicolumn{2}{c}{ T offset } \\
\cline { 2 - 8 } & $q 1 c$ & $q 2 c$ & $q 1 c$ & $q 2 c$ & $q 1 c$ & $q 2 c$ & $q 1 c$ & $q 2 c$ \\
\hline $\begin{array}{c}\text { Total } \\
\text { beat }\end{array}$ & 472 & N.A. & 512 & 401 & 512 & 400 & 512 & 400 \\
TP & 450 & N.A. & 511 & 400 & 465 & 367 & 442 & 348 \\
FP & 19 & N.A. & 1 & 1 & 31 & 22 & 39 & 36 \\
FN & 3 & N.A. & 0 & 0 & 16 & 11 & 31 & 16 \\
ACC & 95.34 & N.A. & 99.8 & 99.75 & 90.82 & 91.75 & 86.33 & 87 \\
Se & 99.34 & N.A. & 100 & 100 & 96.67 & 97.09 & 93.45 & 95.6 \\
PPV & 95.95 & N.A. & 99.8 & 99.75 & 93.75 & 94.35 & 91.89 & 90.63 \\
\hline
\end{tabular}

*Abbreviation: N.A. - not available

\section{Conclusion}

In this study, an efficient and simple ECG delineation algorithm that instantaneously delineate the significant ECG characteristic points were proposed. The proposed algorithm is based on discrete wavelet transform and moving window average techniques which have low 
computational burden. The implementation of decomposition and reconstruction techniques allows the QRS complex and $\mathrm{P}$ and $\mathrm{T}$ waves to be separated and isolated according to their frequency range. This enables the delineation of $\mathrm{P}$ and $\mathrm{T}$ wave to be completed without the need to introduce the QRS removal method and further avoid the miss recognition of $\mathrm{R}$ peak and high $\mathrm{T}$ peak. The proposed algorithm with the isolated $\mathrm{T}$ wave signal also enable the accurate delineation of inverted $\mathrm{T}$ peak that further improve the $\mathrm{T}$ wave delineation performance. Moreover, the wrong or miss delineation of current heartbeat would not influence the delineation of next heartbeat as the delineation is achieved through windowing approach based on two moving window average. The proposed algorithm having low computational cost while achieving adequate delineation results for the significant ECG characteristic points. It is believed that the efficient and low computational instantaneous ECG delineation algorithm is crucial as it plays a pivotal role in the diagnosis of cardiovascular diseases and assisting the clinical practitioner in saving the patient's life nowadays.

\section{Acknowledgments}

This research is supported and funded by Ministry of Higher Education (MoHE) Trans-Disciplinary Research Grant Scheme (TRGS) with Grant no. TRGS/1/2015/UTM/02/3/3 (UTM vote no. R.J130000.7845.4L842) and Universiti Teknologi Malaysia Research University Grant with Vote No. Q.J130000.2545.16H83. The researcher is under sponsorship of Universiti Teknologi Malaysia through Zamalah scholarship.

\section{References}

[1] D. Pandit et al., "Noise reduction in ECG signals using wavelet transform and dynamic thresholding", in Emerging Trends in Neuro Engineering and Neural Computation. Springer. pp. 193-206, 2017.

[2] S. Qureshi, et al., "Noise reduction of electrocardiographic signals using wavelet transforms." Elektronika ir Elektrotechnika, vol. 20, no. 4, pp. 39-42, 2014.

[3] R. Kemppainen, "ECG parameters in short-term prediction of ventricular arrhythmias," 2012.

[4] J. Karjalainen, A. Reunanen, P. Ristola, and M. Viitasalo, "QT interval as a cardiac risk factor in a middle aged population," Heart, vol. 77, no. 6, pp. 543-548, 1997.

[5] L. Fei, and A. J. Camm, "Shortening of the QT interval immediately preceding the onset of idiopathic spontaneous ventricular tachycardia," American Heart Journal, vol. 130, no. 4, pp. 915-917, 1995.

[6] H. Elming, B. Brendorp, L. Køber, N. Sahebzadah, and C. Torp-Petersen, "QTc interval in the assessment of cardiac risk," Cardiac Electrophysiology Review, vol. 6, no.3, pp. 289-294, 2002.

[7] S. Banerjee, R. Gupta, and M. Mitra. "Delineation of ECG characteristic features using multiresolution wavelet analysis method." Measurement, vol. 45, no. 3, pp. 474-487, 2012.

[8] K. Friganovic et al. "Optimizing the detection of characteristic waves in ECG based on processing methods combinations." IEEE Access vol. 6, pp. 50609-50626, 2018.

[9] A. Lyon et al. "Computational techniques for ECG analysis and interpretation in light of their contribution to medical advances." Journal of The Royal Society Interface, vol.15, no. 138, Aug 2017.

[10]P. Laguna, R. G. Mark, A. L. Goldberger, G. B. Moody, “A database for evaluation of algorithms for measurement of QT and other waveform intervals in the ECG." Computers in Cardiology vol. 24, pp. 673-676, 1997.

[11] A. L. Goldberger, L. AN. Amaral, L. Glass, J. M. Hausdorff, P. C. h. Ivanov, R. G. Mark, J. E. Mietus, G. B. Moody, C. K. Peng, H. E. Stanley, "PhysioBank, PhysioToolkit, and PhysioNet: components of a new research resource for complex physiologic signals." Circulation, vol. 101, no. 23, pp. 215-220, 2000.

[12]S. Jain and M. P. Parsai, "Comparative analysis of various wavelet families used for R-wave detection of ECG waveforms." International Journal of Innovative Research in Science Engineering and Technology, vol. 2, no. 10, pp. 5177-5188, 2013.

[13]S. Z. Mahmoodabadi, A. Ahmadian, M. D. Abolhasani, M. Eslami, and J. H. Bidgoli, "ECG feature extraction based on multiresolution wavelet transform." IEEE Engineering in Medicine and Biology, pp. 3902-3905, January 2006.

[14]S. Pal and M. Mitra, "Detection of ECG characteristic points using multiresolution wavelet analysis based selective coefficient method." Measurement, vol. 43, no. 2, pp. 255$261,2010$.

[15]C. Watford, "Understanding ECG filtering", EMS 12-Lead, March 2014.

[16]M. Elgendi, M. Marianna, and A. Derek, "A proof-ofconcept study: Simple and effective detection of $\mathrm{P}$ and $\mathrm{T}$ waves in arrhythmic ECG signals." Bioengineering, vol. 3, no. 4, pp. 26, 2016.

[17]M. Elgendi, "Fast QRS detection with an optimized knowledge-based method: Evaluation on 11 standard ECG databases." PloS one, vol. 8, no. 9, 2013.

[18]D. Sadhukhan, and M. Mitr, "ECG denoising and delineation using multiresolution discrete wavelet transform." Canadian Journal on Technology Innovation, vol, 1, pp. 197-208, 2014.

[19]S. Banerjee, R. Gupta and M. Mitra, "Delineation of ECG characteristic features using multiresolution wavelet analysis method." Measurement, vol. 45, no. 3, pp. 474-487, 2012.

[20]ANSI/AAMI EC57, "Testing and reporting performance results ofcardiac rhythm and ST segment measurement algorithms." Association for the Advancement of Medical Instrumentation, Arlington, 2012.

Address for correspondence:

Yuan Wen Hau

UTM-IJN Cardiovascular Engineering Centre,

School of Biomedical Engineering and Health Sciences, Faculty of Engineering, Universiti Teknologi Malaysia. 81310 UTM Johor Bahru, Johor. Malaysia.

hauyuanwen@biomedical.utm.my 